\title{
Laparoscopic Sigmoidectomy with Preservation of the Superior Rectal Artery: Usefulness of Bipolar Scissors
}

\author{
Tomoyuki Wakahara, Akihiro Toyokawa, Masahide Awadu, Tsuyoshi Takahashi, \\ Shinobu Tsuchida and Yasuhisa Hasegawa \\ Department of Surgery, Yodogawa Christian Hospital
}

\begin{abstract}
In D3 sigmoidectomy, the root of the inferior mesenteric artery (IMA) is usually divided. However, this sometimes causes insufficient blood supply at the anastomosis, leading to anastomotic leakage. We perform superior rectal artery (SRA)-preserving laparoscopic sigmoidectomy for proximal sigmoid colon cancer to preserve the blood supply to the anastomotic site. The vascular sheath along the IMA and the SRA were peeled off together with the lymph nodes using bipolar scissors, then the arteries feeding the tumor were divided at the bifurcation from the SRA. Forty patients underwent this operation, and there were no cases of intraoperative morbidity and none required conversion to laparotomy. There were 2 cases of bowel obstruction, 2 of pneumonia, 1 of pulmonary embolism, and 1 of wound infection; however, there were no cases of anastomotic leakage. Laparoscopic D2 or D3 sigmoidectomy with preservation of the SRA can be safely conducted with our technique using bipolar scissors.
\end{abstract}

Key words: laparoscopic sigmoidectomy, superior mesenteric artery, bipolar scissors

\section{Introduction}

For sigmoid colon cancer, the root of the inferior mesenteric artery (IMA) is usually divided to perform D3 lymph node dissection. However, Dworkin

Received: August 5, 2013/Accepted: December 13, 2013 Correspondence to: Tomoyuki Wakahara Department of Surgery, Yodogawa Christian Hospital, 1-7-50, Kunijima, Higashi Yodogawa Ku, Osaka, 533-0024, Japan et al. ${ }^{1)}$ and Seike et al. ${ }^{2)}$ reported a significant reduction of blood flow in the proximal limb after ligation of the IMA at its origin. Blood supply to the anastomotic site is an important factor for healing ${ }^{3,4}$. Preservation of the superior rectal artery (SRA) can preserve the blood supply for the distal sigmoid colon and the upper rectum after sigmoidectomy, which may decrease the incidence of anastomotic leakage.

Several hemostatic devices have recently been developed and have contributed significantly to progress in laparoscopic surgery methods. Ultrasonic shears are one of the instruments commonly used in laparoscopic surgery. When using the device, the vibrating blade of the device must be confirmed to be separated from blood vessels or viscera. A bipolar vessel-sealing device is also frequently used; however, the thick and dull jaws of the device are not suitable for fine manipulation-such as dissecting lymph nodes surrounding blood vessels. On the other hand, the blades of bipolar scissors are relatively fine and suitable for lymph node dissection, and cutting and coagulation can be performed simultaneously without concern for the cavitation that is specific to ultrasonic shears, though they may have inferior hemostatic ability to the previous 2 devices.

Here we report our technique of SRA-preserving sigmoidectomy using bipolar scissors and the shortterm results of the procedure.

\section{Materials and Methods}

Patients with proximal sigmoid colon cancer were candidates for the operation. The location of the lesion was assessed by performing a barium study of the colon, and the oral half of the sigmoid colon was defined as the proximal sigmoid colon. 


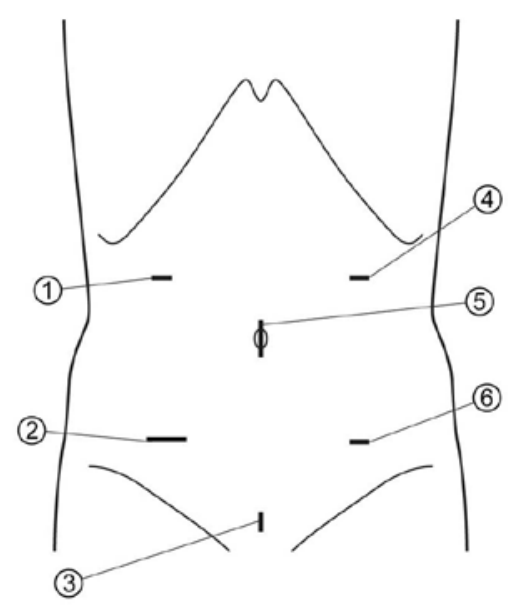

Fig. 1 The location of the trocars.

(1) $5 \mathrm{~mm}$ (laparoscope) (2) $12 \mathrm{~mm}$ (3) $5 \mathrm{~mm}$

(4) $5 \mathrm{~mm}$ (5) $12 \mathrm{~mm}$ (6) $5 \mathrm{~mm}$

The patients were laid in the head-down and right-side-down position with $\mathrm{CO}_{2}$-induced pneumoperitoneum. The laparoscope was inserted through a $5-\mathrm{mm}$ trocar placed in the upper right quadrant. Two $12-\mathrm{mm}$ trocars were placed infraumbilically and in the lower right quadrant for the operator, and $25-\mathrm{mm}$ trocars were inserted in the left lateral lower and upper quadrant for the assistant (Fig. 1 ). An additional 5-mm trocar was inserted into the suprapubic region if needed. After the small intestine was placed in a cephalad orientation to expose the operative field, including the root of the IMA, the assistant held the pedicle of the SRA and the mesosigmoid ventrally. The surgery was then begun with an incision of the right surface of the sigmoid mesocolon using an electrocautery spatula.

The incision was elongated cephalad and caudally, expanding the dissection layer just beneath the mesorectal fascia and preserving the hypogastric nerve. The incision in the serosa and the mobilization of the mesosigmoid continued to the root of the IMA. Then, using a sealing device, lymph nodes and adipose tissue at the root of the IMA were dissected and the wall of the artery was exposed. Lymph nodes were then detached from the artery with bipolar scissors sliding along the arterial wall (Fig. 2a), peeling off the vascular sheath along the
IMA and the SRA, and exposing the bifurcation of the left colic artery (LCA) and the sigmoid arteries (Fig. 2b ). Small blood vessels surrounding the arteries or vasa vasorum were coagulated, and the sheath was cut with bipolar scissors if necessary. Exposition of the inferior mesenteric vein and dissection of lymph nodes surrounding the vein could be also performed in the same manner using bipolar scissors.

The sigmoid and descending mesocolon were then mobilized in a medial to lateral approach, maintaining the former dissecting layer to avoid injury to the gonadal vessels and the ureter. After dissecting the lateral attachment of the sigmoid and descending colon, the precise location of the tumor and the distribution of the arteries were confirmed to determine which artery to divide. The arteries feeding the tumor were clipped and divided at the bifurcation from the SRA (Fig. 2c).

Resection of the colon and anastomosis were performed extracorporeally after removing the mobilized sigmoid colon through a minilaparotomy located in the infraumbilical or left lumbar region (Fig. 3 ).

\section{Results}

From April 2008 to May 2011, 40 patients with proximal sigmoid colon cancer underwent this operation. Patient characteristics and the postoperative results are listed in Table $\mathbf{1}$. The mean quantity of blood loss was $33.0 \mathrm{~g}$, and no case required blood transfusion. The mean operative time was 285 minutes. There were no cases of intraoperative morbidity and none required conversion to laparotomy. The postoperative morbidity rate was $15.0 \%$. There were 2 cases of bowel obstruction, 2 of pneumonia, 1 of pulmonary embolism, and 1 of wound infection; however, there were no cases of anastomotic leakage.

Three cases of recurrence were noted. One case was of a patient with Stage III a disease (Japanese Classification of Colorectal Carcinoma, The $7^{\text {th }}$ Edition) in whom peritoneal dissemination was observed 273 days after surgery, the second case was of a patient also with Stage III a disease who developed lung metastasis 715 days after surgery, and the third case was of a patient with Stage II disease who developed abdominal wall metastasis 757 days after surgery. Recurrence-free survival (RFS) and 

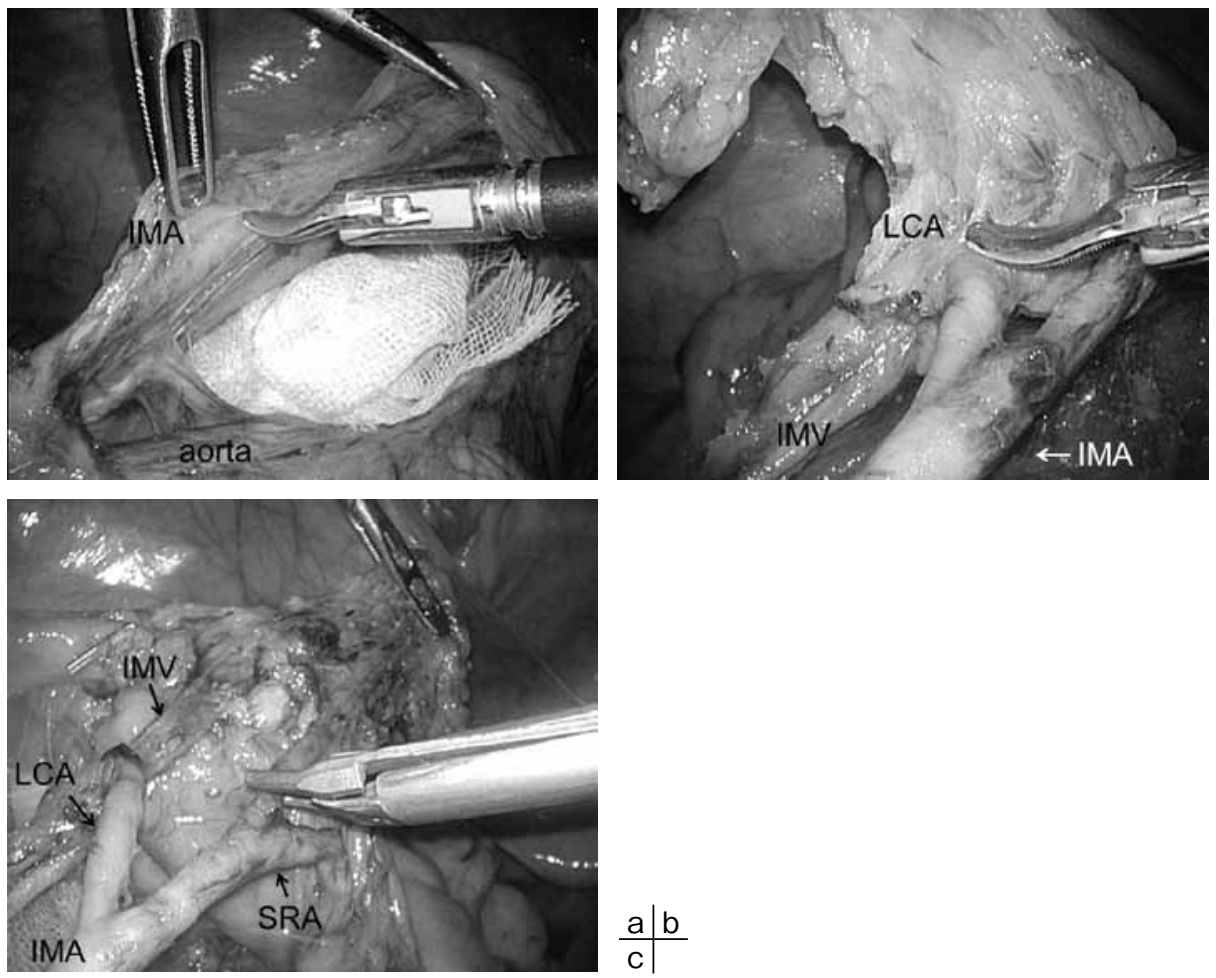

Fig. 2 a: After lymph nodes at the root of the IMA were dissected and the wall of the artery was exposed, lymph nodes were detached from the artery with bipolar scissors sliding along the arterial wall, peeling off the vascular sheath along the IMA and the SRA. b: The bifurcation of the SRA and the LCA was exposed.

c: After the skeletonization of the arteries and the mobilization of the sigmoid and descending colon, the arteries feeding the tumor were clipped and divided at the bifurcation from the SRA.

overall survival (OS) were estimated in the 36 cases, excluding the 4 cases of Stage IV disease with non-curative resection, using the Kaplan-Meier method. The median follow-up period was 1,287 days, the 3 -year RFS rate was $87.5 \%$, and the 3 -year OS rate was $96.9 \%$. Statistical analysis was performed using JMP version 8 software (JMP, Chicago, IL, USA).

\section{Discussion}

Anastomotic leakage is a serious postoperative morbidity that is reported to occur in $3.6 \%$ to $6 \%$ of patients after colorectal surger $\mathrm{y}^{5-9)}$, and is associated with prolonged hospital stays, delays in starting postoperative adjuvant therapy, and often requires diverting stoma. Anastomotic leakage also increases the postoperative mortality rate. Some authors advocate that it leads to a significant reduction in long-term survival ${ }^{5,8,10-12)}$. A variety of factors, including low serum albumin level, male gender, the duration of surgery, steroid use, postoperative blood transfusion, and anterior resection, have been identified as risk factors for anastomotic leakage after colorectal surgery ${ }^{5,9)}$; however, blood supply to the intestine surrounding the anastomosis is also an important factor ${ }^{3,4}$. In cases of benign disease, several authors have reported the advantage of preservation of the SRA in preventing anastomotic leakage ${ }^{13,14)}$. In our series, postoperative morbidity occurred in $15.0 \%$ of patients; but, there was no anastomotic leakage. Preservation of the SRA and the left colic artery (LCA) may have improved the blood flow of the distal and the proximal limb of the sigmoid colon, respectively, resulting in a reduction of anasto- 


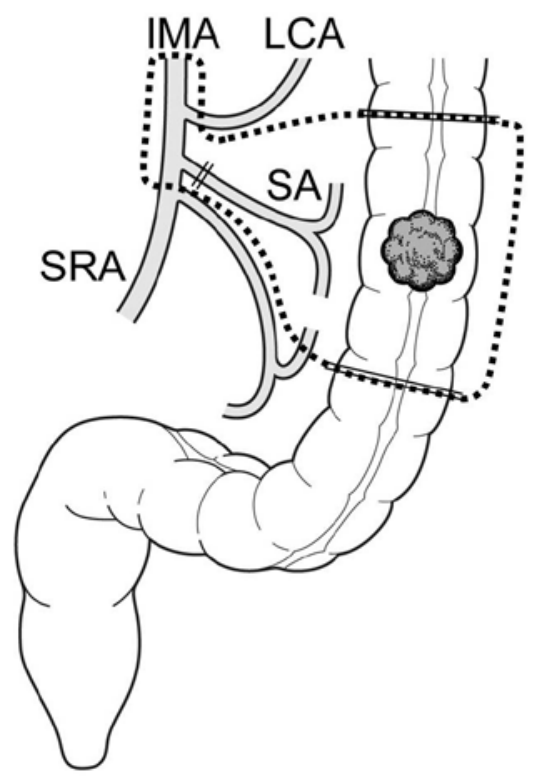

Fig. 3 An example of the SRA-preserving sigmoidectomy. The arteries feeding the tumor were confirmed after mobilization of the sigmoid colon and divided at the bifurcation from the SRA. In this scheme, only the $1^{\text {st }}$ sigmoid artery (SA) was divided. The dotted line shows the extent of D3 lymph node dissection.
Table 1 Characteristics of the participating patients and results of the operation

\begin{tabular}{|c|c|}
\hline $\mathrm{n}$ & 40 \\
\hline Mean age (range) & $65.9(39-88)$ \\
\hline \multicolumn{2}{|l|}{ Gender } \\
\hline Male & 22 \\
\hline Female & 18 \\
\hline \multicolumn{2}{|l|}{ ASA score } \\
\hline 1 & 9 \\
\hline 2 & 28 \\
\hline 3 & 3 \\
\hline Mean BMI (range) & $23.8(17.7-30.6)$ \\
\hline \multicolumn{2}{|l|}{ Tumor depth } \\
\hline $\mathrm{pM}$ & 3 \\
\hline pSM & 10 \\
\hline pMP & 5 \\
\hline pSS & 16 \\
\hline pSE & 5 \\
\hline pSI & 1 \\
\hline Mean tumor size $(\mathrm{mm})$ (range) & $39.6(5-80)$ \\
\hline \multicolumn{2}{|l|}{ Node involvement } \\
\hline pNO & 29 \\
\hline pN1 & 10 \\
\hline pN2 & 1 \\
\hline \multicolumn{2}{|l|}{ pStage } \\
\hline 0 & 3 \\
\hline 1 & 14 \\
\hline 2 & 11 \\
\hline 3 & 8 \\
\hline 4 & 4 \\
\hline Blood Loss (g) & $33.0(5-170)$ \\
\hline Operative Time (minutes) & $285(183-410)$ \\
\hline Conversion to Open Surgery & 0 \\
\hline Harvested Lymph Nodes & $15.7(3-37)$ \\
\hline Intraoperative Morbidity & 0 \\
\hline Postoperative Morbidity & 6 \\
\hline bowel obstruction & 2 \\
\hline pneumonia & 2 \\
\hline pulmonary embolism & 1 \\
\hline wound infection & 1 \\
\hline Mortality & 0 \\
\hline Reoperation & 0 \\
\hline
\end{tabular}

ASA American Society of Anesthesiologists, BMI body mass index motic leakage.

In patients with malignant diseases in which lymph node dissection is important, the root of the IMA is usually divided when D3 lymph node dissection is performed, and preservation of the IMA or SRA may lead to insufficient lymph node dissection. Some authors advocate the survival benefit of ligating the IMA at its origin ${ }^{15}$; but, there is insufficient evidence to perform high ligation as a standard procedure $^{16)}$. In our series, the mean number of harvested lymph nodes was 15.7 in sigmoidectomy with D2 or D3 lymph node dissection, which is thought to be sufficient as a cancer surgery. The long-term survival of sigmoid colon cancer patients after SRApreserving sigmoidectomy has not yet been reported, and should be examined in a prospective randomized study comparing sigmoidectomy with and without SRA preservation.

An advantage of using bipolar scissors to dissect lymph nodes surrounding blood vessels is that electric cauterization can be safely performed even when the blades of the bipolar scissors are in contact with the vessels, whereas coagulation with a monopolar electrocautery spatula can cause injury to the blood vessels if it touches the vessels. Thus, the electrocautery spatula must be used with strict attention to confirming that it does not contact the vessels. Hemorrhage from small blood vessels adjacent to the arteries or vasa vasorum can be difficult to address using an electrocautery spatula; however, coagulation with bipolar scissors can be performed even while it touches the blood vessels. We had no intraoperative morbidity in relation to this technique. The patients were followed at intervals of 3 months, and a computer tomography scan was performed every 6 months. No abnormalities, including aneurysm formation, were caused by this technique.

In conclusion, SRA-preserving sigmoidectomy 


\section{SRA-Preserving Sigmoidectomy}

with D2 or D3 lymph node dissection can be safely conducted with our method using bipolar scissors without intraoperative hemorrhagic complications or postoperative aneurysm formation caused by burning of the arterial wall. However, the long-term outcome of this procedure has yet to be evaluated.

\section{References}

1) Dworkin MJ, Allen-Mersh TG: Effect of inferior mesenteric artery ligation on blood flow in the marginal artery-dependent sigmoid colon. J Am Coll Surg $183: 357-360,1996$

2) Seike K, Koda K, Saito N, et al : Laser doppler assessment of the influence of division at the root of the inferior mesenteric artery on anastomotic blood flow in rectosigmoid cancer surgery. Int J Colorectal Dis 22:689-697, 2007

3) Shikata J, Shida T, Satoh S, et al : The effect of local blood flow on the healing of experimental intestinal anastomoses. Surg Gynecol Obstet 154:657-661, 1982

4) Foster ME, Lancaster JB, Leaper DJ : Leakage of low rectal anastomosis (An anatomic explanation?). Dis Colon Rectum $27: 157-158,1984$

5) Boccola MA, Buettner PG, Rozen WM, et al : Risk factors and outcome for anastomotic leakage in colorectal surgery: A single-institution analysis of 1,576 patients. World J Surg $35: 186-195,2011$

6) Lehmann RK, Brounts LR, Johnson EK, et al : Does sacrifice of the inferior mesenteric artery or superior rectal artery affect anastomotic leak following sigmoidectomy for diverticulitis? A retrospective review. Am J Surg 201 : 619-623, 2011

7) Post IL, Verheijen PM, Pronk A, et al : Intraoperative blood pressure changes as a risk factor for anastomotic leakage in colorectal surgery. Int $\mathrm{J}$ Colorectal Dis 27:765-772, 2012

8) Alves A, Panis Y, Trancart D, et al : Factors associated with clinically significant anastomotic leakage after large bowel resection: multivariate analysis of 707 patients. World J Surg 26:499-502, 2002

9) Suding P, Jensen E, Abramson MA, et al : Definitive risk factors for anastomotic leaks in elective open colorectal resection. Arch Surg 143:907-912, 2008

10) Makela JT, Kiviniemi H, Laitinen $S$ : Risk factors for anastomotic leakage after left-sided colorectal resection with rectal anastomosis. Dis Colon Rectum $46: 653-660,2003$

11) Law WL, Choi HK, Lee YM, et al: Anastomotic leakage is associated with poor long-term outcome in patients after curative colorectal resection for malignancy. J Gastrointest Surg $11: 8-15,2007$

12) McArdle CS, McMillan DC, Hole DJ : Impact of anastomotic leakage on long-term survival of patients undergoing curative resection for colorectal cancer. Br J Surg $92: 1150-1154,2005$

13) Bergamaschi R, Lovvik K, Marvik R : Preserving the superior rectal artery in laparoscopic sigmoid resection for complete rectal prolapse. Surg Laparosc Endosc Percutan Tech 13:374-376, 2003

14) Tocchi A, Mazzoni G, Fornasari V, et al : Preservation of the inferior mesenteric artery in colorectal resection for complicated diverticular disease. Am J Surg 182:162-167, 2001

15) Kanemitsu $Y$, Hirai $T$, Komori $K$, et al : Survival benefit of high ligation of the inferior mesenteric artery in sigmoid colon or rectal cancer surgery. $\mathrm{Br} \mathrm{J}$ Surg $93: 609-615,2006$

16) Lange MM, Buunen M, van de Velde CJ, et al : Level of arterial ligation in rectal cancer surgery : low tie preferred over high tie. A review. Dis Colon Rectum $51: 1139-1145,2008$ 